\title{
POCAM in the IceCube Upgrade
}

\section{The IceCube Collaboration}

(a complete list of authors can be found at the end of the proceedings)

E-mail: nikhita.khera@tum.de, felix.henningsen@tum.de

The IceCube Neutrino Observatory at the geographic South Pole instruments a gigaton of glacial Antarctic ice with over 5000 photosensors. The detector, by now running for over a decade, will be upgraded with seven new densely instrumented strings. The project focuses on the improvement of low-energy and oscillation physics sensitivities as well as re-calibration of the existing detector. Over the last few years we developed a Precision Optical Calibration Module (POCAM) providing self-monitored, isotropic, nanosecond, light pulses for optical calibration of large-volume detectors. Over 20 next-generation POCAMs will be calibrated and deployed in the IceCube Upgrade in order to reduce existing detector systematics. We report a general overview of the POCAM instrument, its performance and calibration procedures.

Corresponding authors: Nikhita Khera ${ }^{1 *}$, Felix Henningsen ${ }^{1,2}$

${ }^{1}$ Technical University of Munich, James-Frank-Str. 1, 85748 Garching, Germany

${ }^{2}$ Max Planck Institute for Physics, Föhringer Ring 6, 80805 München, Germany

${ }^{*}$ Presenter

$37^{\text {th }}$ International Cosmic Ray Conference (ICRC 2021)

July 12th - 23rd, 2021

Online - Berlin, Germany 


\section{POCAM and its housing}

The Precision Optical Calibration Module (POCAM) [1] [2] is designed to be a high precision, self-monitoring, isotropic, nanosecond, multi-wavelength calibration light source for a precise measurement of the energy scale and resolution within the IceCube Upgrade [3]. This instrument, as shown in Figure 1, has a cylindrical titanium housing, which hosts the circuit boards with light flashers, read out electronics and a PTFE integrating sphere, encapsulated finally by a BK-7 glass hemisphere. Two such identical hemisphere assemblies are on either side of the titanium cylinder. The analog PCB has six optical emitters - three light emitting diodes (LEDs) (365nm, 405nm, 465 $\mathrm{nm}$ ) and three laser diodes (LDs) $(405 \mathrm{~nm}, 455 \mathrm{~nm}, 520 \mathrm{~nm})$ - for flashing. On top of these, integrated on the aperture disk, are two sensors - a silicon photomultiplier (SiPM) and a photodiode (PD) installed for self-monitoring. The PTFE integrating sphere is responsible for making the light pulse isotropic [4] [5].
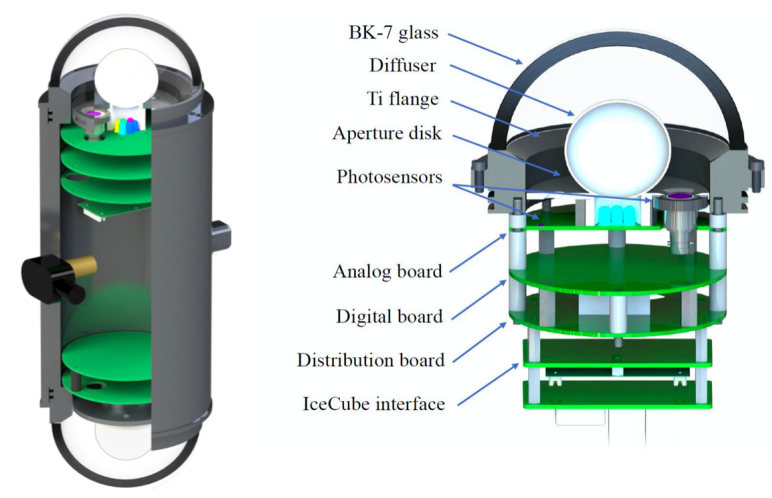

Figure 1: POCAM module and POCAM hemisphere assembly [4]

To verify the performance and stability of the POCAM, the housing was tested in a high pressure chamber at Nautilus and temperature testing was done by placing it in a freezer at $-55{ }^{\circ} \mathrm{C}$ for five weeks. Vibration and shock tests on the POCAM stack (i.e. the hemisphere assembly as shown in Figure 1 without the glass) were performed and passed at the IABG test site in Ottobrunn, Germany. After the development of the POCAM started in the late 2014, a first protype of the device was successfully deployed in the GVD neutrino telescope at Lake Baikal, Russia in 2017 [1]. An improved second iteration of the device was then installed within the STRAW experiment [6] in the North East Pacific deep sea in the year 2018. After these succesfull deployments, this latest iteration of the POCAM that we report about in this paper, with significant improvements and changes, is now being developed in the scope of the IceCube Upgrade project [4] [7].

\section{Light Emitters}

The analog boards in the POCAM electronics incorporate six light emitters out of which three are LEDs and three are LDs. Further, two Kapustinsky [8] and two LD-type LD drivers [9] are responsible for the driving of light pulses from these emitters (Figure 2). The purpose to keep two of each type of drivers is to not only allow redundancy but also a freedom to selectively drive 
different pulsers for different flasher wavelengths. We have two LD type drivers as well as a fast and a default Kapustinsky circuit.
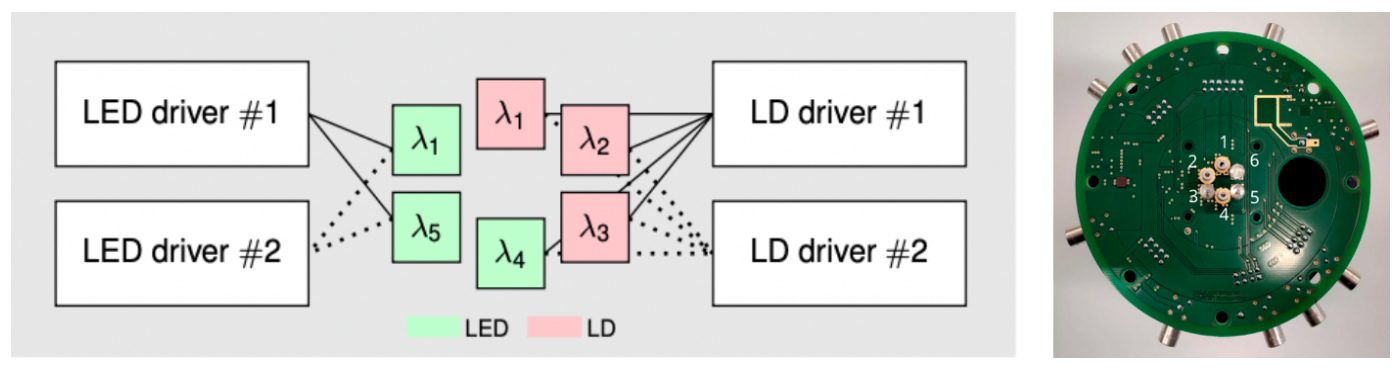

Figure 2: Layout of LEDs, LDs and pulse drivers in the POCAM (left) [4], LEDs and LDs on the analog board (right)

The Kapustinsky circuit is developed for LEDs and makes use of a relatively simple and pre-proven circuitry as shown in the Figure 3. The circuit operates on negative bias voltage and produces a pulse when triggered with a square pulse signal. The pulse properties can be modified by controlling the values of capacitor $\mathrm{C}$ and the inductance $\mathrm{L}$ and the pulse width scales qualitatively with $\sqrt{L C}$.
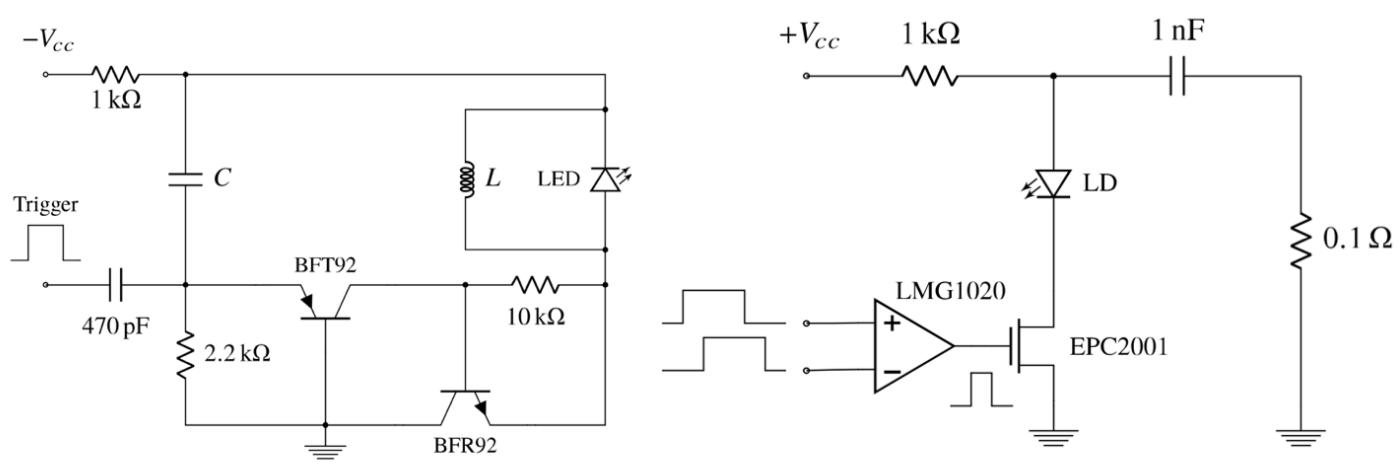

Figure 3: Schematic of the Kapustinsky circuit (left) and the LD type driver circuit (right) [4]

For the POCAM application in IceCube Upgrade, the Kapustinsky circuit is configured to drive the $405 \mathrm{~nm}$ and $465 \mathrm{~nm}$ LEDs. Each of these LEDs can select a fast (L, C - $22 \mathrm{nH}, 100 \mathrm{pF}$ ) or default (L,C - $22 \mathrm{nH}, 1.2 \mathrm{nF}$ ) Kapustinsky driver configuration with different pulse widths and light yields.

The third and the fourth driver on the analog board are the LD type drivers which are configured to drive a $365 \mathrm{~nm}$ LED and three LDs at $405 \mathrm{~nm}, 455 \mathrm{~nm}$ and $520 \mathrm{~nm}$. These drivers are equipped with fast high-current switching GaN-FETS (Gallium-Nitride field effect transistors). The picosecond switching of these GaN-FETs allow the possibility of adjustable pulse widths and significantly more light output. The circuitry of the LD driver is also shown in the Figure 3. The circuit operates on positive bias voltage and produces a pulse proportional to the input signal. 


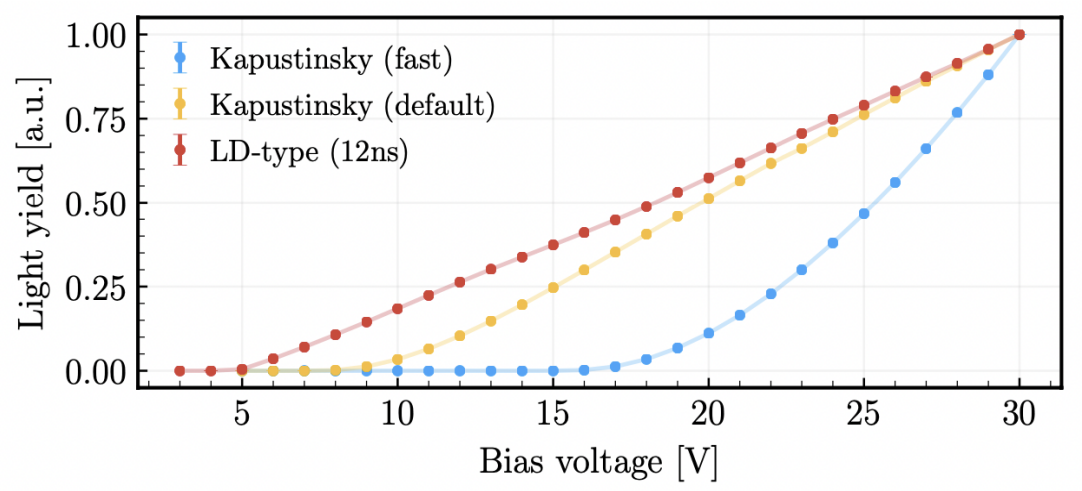

Figure 4: Intensity yield (measured with an external photodiode) as a function of voltage for all the drivers at $405 \mathrm{~nm}$. [4]

Both flasher circuits perform in different regions of the dynamic range of the POCAM. The intensity behaviour of all driver circuits (observed using an external photodiode) and their respective time profiles (using an avalanche photodiode) were measured for the $405 \mathrm{~nm}$ LED. These can be seen in Figures 4 and 5 respectively.

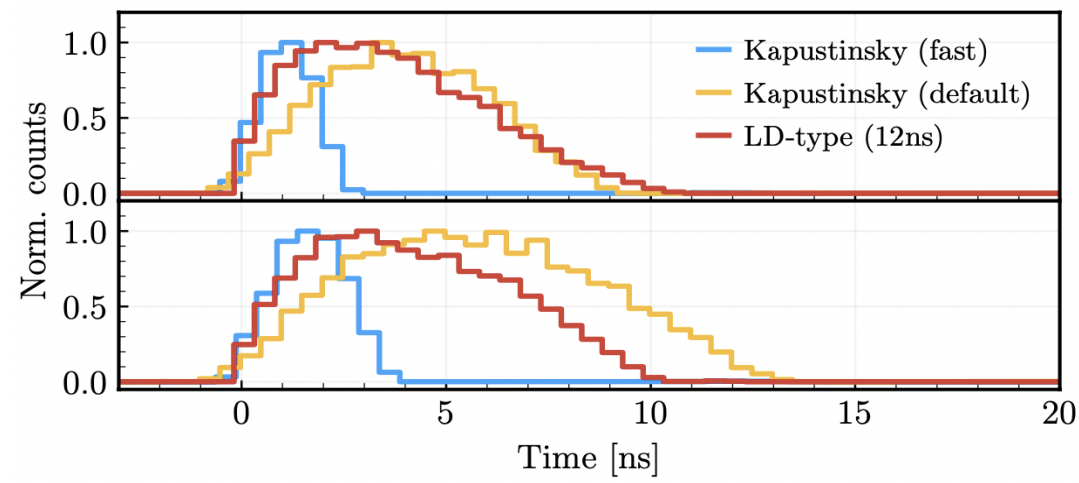

Figure 5: Time profiles (measured using an avalanche photodidoe) at minimal-working (top) and maximal (bottom) applied bias voltage for all the drivers at $405 \mathrm{~nm} .[4]$

As visible in the voltage vs. light yield plot (Figure 4), the fastest Kapustinsky has a significantly late light onset, followed by the default Kapustinsky and then the LD driver which shows linear intensity behaviour. The timing profiles (Figure 5) for the Kapustinskies have been fine tuned to an FWHM of $1-3 \mathrm{~ns}$ for the fast and $4-8 \mathrm{~ns}$ for the default configuration. The LD drivers on the other hand are optimized to generate a wide range of pulse widths (here 12ns).

\section{Self-monitoring sensors}

In order for the POCAM to self monitor the light output per pulse and correct for any intensity fluctuations, two sensors- a SiPM and a photodiode (PD) - are embedded into the aperture disk of the POCAM. The SiPM intends to work in the low intensity regime and makes use of an FPGA discriminator, the signal from which is then fed into a time-digital-converter (TDC) to 
determine the time-over-threshold (ToT). Whereas the photodiode is responsible for high intensity light measurements and makes use of a transimpedence amplifier (TIA), followed by secondary high gain and low gain amplifiers, which provides a voltage amplitude proportional to the measured charge of the PD.
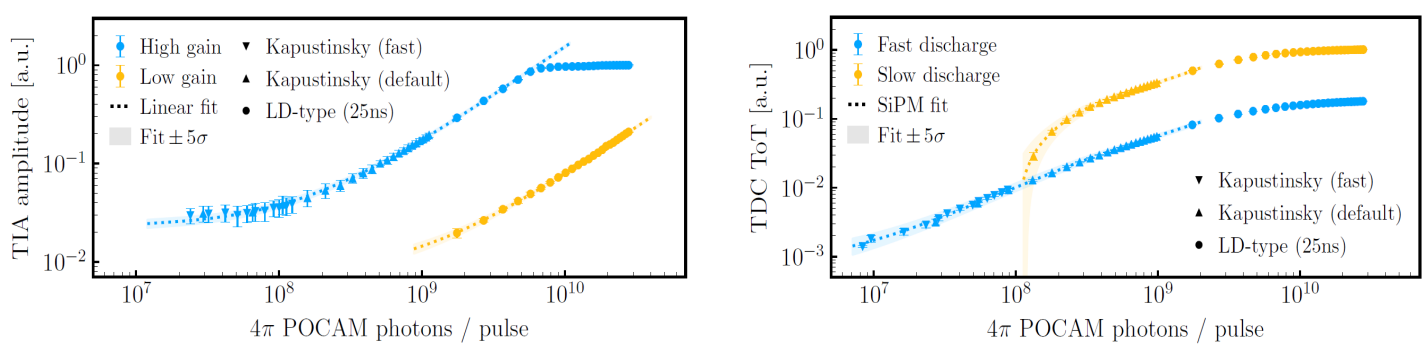

Figure 6: (Left) Readout response of the photodiode for all the drivers at $405 \mathrm{~nm}$.(Right) Readout response of the SiPM for all the drivers at $405 \mathrm{~nm}$.(SiPM over-voltage 5V). [4]

The optimized response behaviour of both these sensors as a function of the POCAM light emission is shown in Figure 6. As expected, the PD showed linear behaviour across all its measurable range. The curve shows saturation at the high gain channel but as can be seen is compensated by the low gain channel. Also the expected saturation curve of the SiPM can easily be fit with appropriate exponential functions [4].

\section{Calibration setups}

To characterize the flashers and the emission profile of both the hemispheres of the POCAM, two calibration setups have been developed.

\subsection{Flasher calbration setup}

In the first setup (Figure 7), to calibrate the flashers, a dark box has been built to house sensors which measure characteristics of the light from the POCAM light source (or any light source), excluding/cutting off the light from the surroundings/natural light. These sensors are each coupled to one end of a 4-to-1 fan-out fiber. This dark box houses four sensors. One, a photodiode, which records the light intensity and is read out by a Keithley 6485 pico-ammeter. Next, a photomultiplier tube (PMT), which also records light intensity and the PMT pulses are further recorded with the help of a digital oscilloscope. Third, an $A P D$, which uses time-correlated single photon counting to measure the pulse time profile. Moreover, the APD is also equipped with a controllable neutral density filter wheel to achieve low occupancies of below $10 \%$ which is necessary to provide proper single-photon sampling of the time profile. The APD signal is fed into a high-precision TDC. Finally, a spectrometer is also installed, which directly records and outputs the spectrum via serial command.

All of these sensors are connected to the necessary power supplies and other peripheral electronics, and are further controlled by a dedicated computer running all the necessary software. To begin, the POCAM is first put inside the freezer and the fan-out fiber is coupled to the teflon sphere. This system then performs automatic temperature scans and measures the pulse properties 
along with the self-monitoring sensor responses. The data generated is then used offline for characterization of individual POCAM hemispheres (upto 3 days per POCAM) giving us fingerprintcharacterized or golden POCAMs.
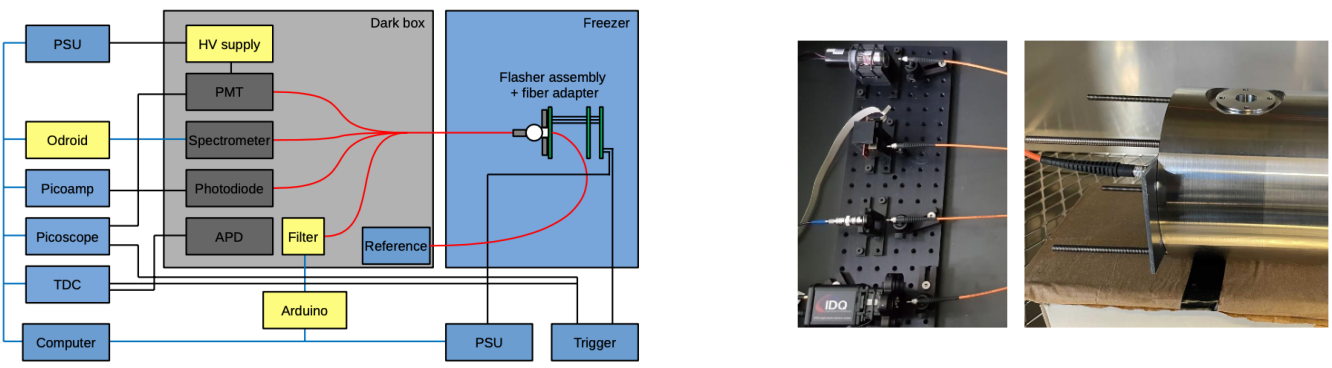

Figure 7: Flasher calibration workflow diagram(left) [4] and real laboratory setup of the dark box - freezer assembly

Nitrogen flushing: To remove further systematic uncertainties resulting from potential fiber coupling changes over the course of cooling and heating, we flush the freezer with nitrogen. Moreover we also monitor a temperature-stabilized reference halogen light source coupled into the integrating sphere using the same type of fiber. A schematic of our current setup is shown in Figure 8. With this setup, we monitored only our reference source, to see any temperature dependent intensity variations. For this purpose, an additional 3-D printed holder for the photodiode is also placed inside the dark box. For this particular procedure, the photodiode needs to be mounted on this holder rather than sit back with the other sensors. It is possible to adjust the position of the holder with the help of screws on the floor of the dark box. The height however is unchangeable because it is set so as to see the light from the light source in the freezer. Carrying out this measurement, the plot in Figure 8 was generated.
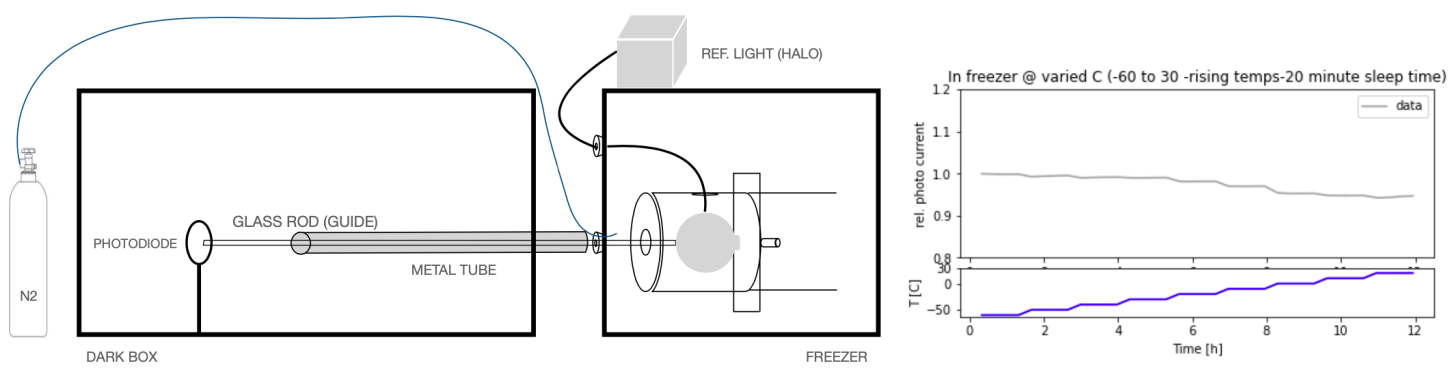

Figure 8: (Left) Schematic of lab setup for reference source monitoring.(Right) Reference source intensity as a function of time. The sleep time here is the duration the freezer takes to gets stable between temperature changes. A quartz glass guide was used to guide the light from the integrating sphere to the sensor

\subsection{Relative isotropy calibration}

The second calibration station is made to measure the emission profile of the POCAM (Figure 9). This setup also consists of a dark box, which has a two-axis rotation stage assembly, where the POCAM hemisphere (i.e the flange, integrating spheres, apperture disk and sensors) can be 
mounted. At the other end of the dark box, just opposite the rotation stage, a photodiode is mounted. The light baffles in between further reduce stray light from reflections off of inner surfaces. After mounting the POCAM hemisphere on the rotation stage, light from the LEDs on the simplified analog board (with a layout identical to the analog board i.e the same led matrix and light emission characteristics) then falls on the PD. A dedicated computer, running all the necessary software, then carries out the azimuth and zenith angles scans, runs the LEDs and measures the intensity seen by the PD. All of this data which is eventually written to file, can then be accessed offline. The rotation of the hemisphere provides a relative characterization of its emission profile and can further be used to calculate the total hemispherical light yield.

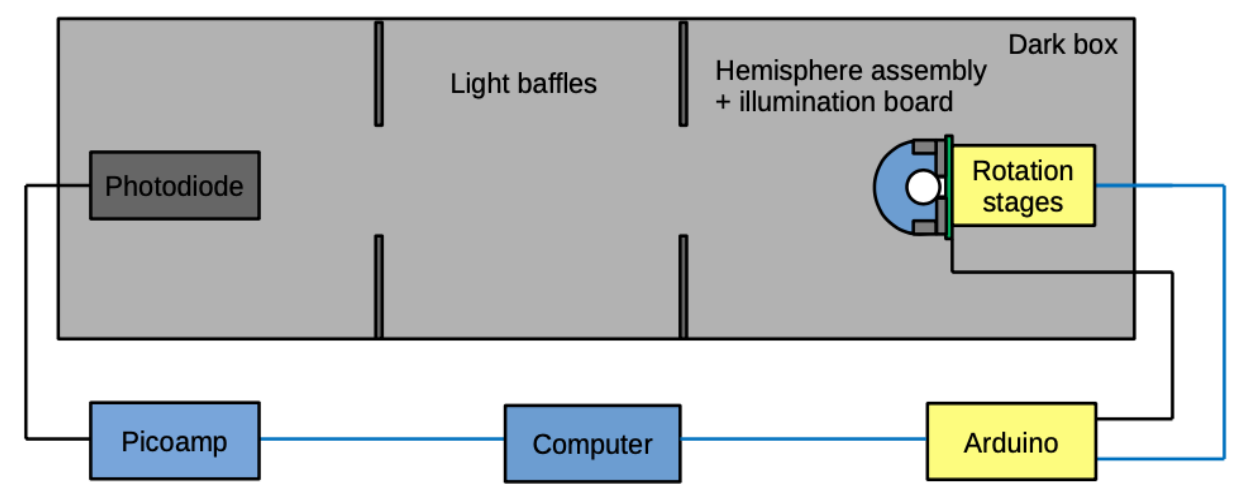

Figure 9: Schematic of the emission profile measurement station [4]

Absolute calibration: After successfully finishing the relative calibration of all POCAM hemispheres, an absolute calibration using a NIST (National Institute for Standards and Technology) calibrated PD is carried out. These absolute intensity scales then act as a reference for self-monitoring data over the course of the operational period of the POCAM and can be used for correcting the instrument emission in-situ using the integrated photosensors. An absolute light yield uncertainty of $4.1 \%$ is anticipated, the dominant uncertainty coming from temperature-dependent fiber coupling $(2 \%)$.

\section{POCAM prototype calibration}

Both flasher and emission profile characterizations were performed on a prototype POCAM before going towards production. The pulser intensity was measured for all driver types, using the $405 \mathrm{~nm}$ emitter (Figure 10). The pulsers show lower intensities at lower temperatures, which could possibly be reasoned by the increased value of the series resistance. The time profile and emission spectrum of the light were also measured but they did not show a significant temperature dependence at high intensities. At lower intensities, however, the time profile plot becomes longer for the LD driver, but it shouldn't be a problem since the LD drivers will be used predominantly for high intensities.

For isotropy measurements, the emission profile of the prototype POCAM hemisphere and then the virtual sum of two hemispheres so as to replicate that of a complete POCAM, is shown via 

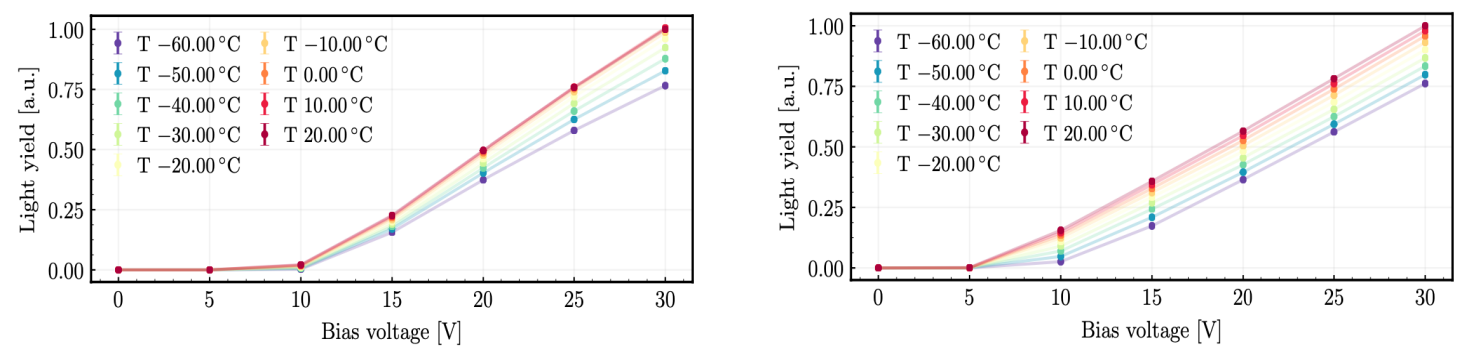

Figure 10: Pulser intensity as a function of voltage at different temperatures for the default Kapustinsky (left) and the LD driver at 25ns (right) [4]

a Mollweide projection in Figure 11. By averaging per LED and all azimuthal angles and using the resulting standard deviation as errors, the isotropy measured shows a $1 \sigma$-error of $1.5 \%$ over the entire zenith range and only $0.4 \%$ between $0-60 \mathrm{deg}$. Estimation of the total $4 \pi$ light yield with $405 \mathrm{~nm}$ LED gives a typical value of $(5.1 \pm 0.4) \times 10^{7}$ photons per pulse for the fast Kapustinsky, $(7.5 \pm 0.6) \times 10^{8}$ photons per pulse for the default Kapustinsky and $(2.4 \pm 0.2) \times 10^{10}$ photons per pulse for the LD type at $25 \mathrm{~ns}$. [4]
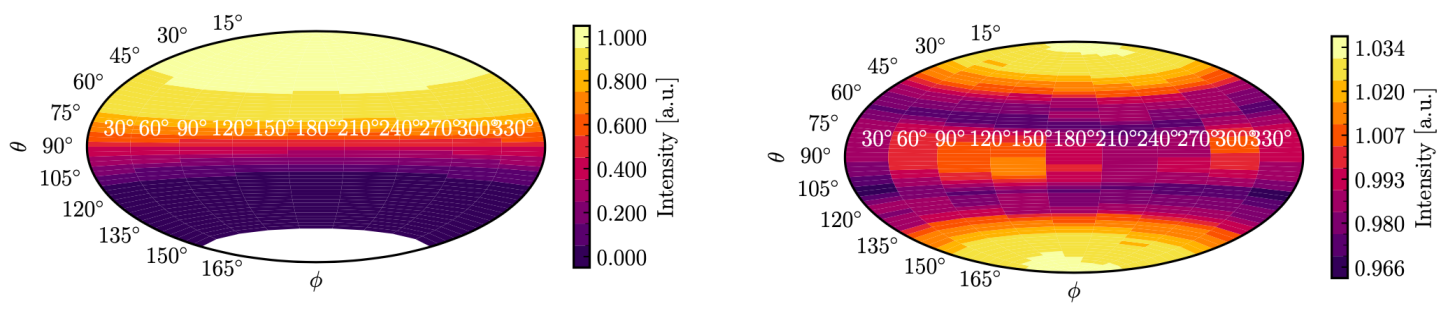

Figure 11: Prototype POCAM single hemisphere emission profile (left) and virtual emission profile of the complete POCAM estimated by overlapping two single hemisphere emissions(right) [4]

\section{References}

[1] IceCube-Gen2 Collaboration, E. Resconi et al., PoS ICRC2017 (2018), 934

[2] IceCube-Gen2 Collaboration, K.Krings, PoS ICRC2015 (2016), 1133

[3] IceCube-Gen2 Collaboration, C. Fruck et al., PoS ICRC2019 (2020), 908

[4] F. Henningsen et al. A self-monitoring precision calibration light source for large-volume neutrino telescopes JINST 15 (2020) no.07, P07031 [arXiv:2005.00778 [astro-ph.IM]]

[5] Berghof Fluoroplastic Technology GmbH, Optical PTFE - The reference for light.

[6] STRAW Collaboration, M. Boehmer et al., JINST 14 (2019) P02013

[7] IceCube-Gen2 Collaboration, A.Ishihara, PoS ICRC2019 (2020), 1031

[8] J. Kapustinsky et al., Nucl. Instr. Meth. Phys. Res. Sec.A 241 (1985) 612 - 613.

[9] EPC. Development Board, EPC9126/EPC9126HC Quick Start Guide, 2019. Rev. 3.0. 


\section{Full Author List: IceCube Collaboration}

R. Abbasi ${ }^{17}$, M. Ackermann ${ }^{59}$, J. Adams ${ }^{18}$, J. A. Aguilar ${ }^{12}$, M. Ahlers ${ }^{22}$, M. Ahrens ${ }^{50}$, C. Alispach ${ }^{28}$, A. A. Alves Jr. ${ }^{31}$, N. M. $\mathrm{Amin}^{42}$, R. An ${ }^{14}$, K. Andeen ${ }^{40}$, T. Anderson ${ }^{56}$, G. Anton ${ }^{26}$, C. Argüelles ${ }^{14}$, Y. Ashida ${ }^{38}$, S. Axani ${ }^{15}$, X. Bai ${ }^{46}$, A. Balagopal V. ${ }^{38}$, A. Barbano ${ }^{28}$, S. W. Barwick ${ }^{30}$, B. Bastian ${ }^{59}$, V. Basu ${ }^{38}$, S. Baur ${ }^{12}$, R. Bay ${ }^{8}$, J. J. Beatty ${ }^{20,21}$, K.-H. Becker ${ }^{58}$, J. Becker Tjus ${ }^{11}$, C. Bellenghi $^{27}$, S. BenZvi ${ }^{48}$, D. Berley ${ }^{19}$, E. Bernardini ${ }^{59,60}$, D. Z. Besson ${ }^{34,61}$, G. Binder ${ }^{8,9}$, D. Bindig ${ }^{58}$, E. Blaufuss ${ }^{19}$, S. Blot ${ }^{59}$, M. Boddenberg ${ }^{1}$, F. Bontempo ${ }^{31}$, J. Borowka ${ }^{1}$, S. Böser ${ }^{39}$, O. Botner ${ }^{57}$, J. Böttcher ${ }^{1}$, E. Bourbeau ${ }^{22}$, F. Bradascio ${ }^{59}$, J. Braun ${ }^{38}$, S. Bron $^{28}$, J. Brostean-Kaiser ${ }^{59}$, S. Browne ${ }^{32}$, A. Burgman ${ }^{57}$, R. T. Burley ${ }^{2}$, R. S. Busse ${ }^{41}$, M. A. Campana ${ }^{45}$, E. G. Carnie-Bronca ${ }^{2}$, C. Chen ${ }^{6}$, D. Chirkin ${ }^{38}$, K. Choi ${ }^{52}$, B. A. Clark ${ }^{24}$, K. Clark ${ }^{33}$, L. Classen ${ }^{41}$, A. Coleman ${ }^{42}$, G. H. Collin ${ }^{15}$, J. M. Conrad ${ }^{15}$, P. Coppin $^{13}$, P. Correa ${ }^{13}$, D. F. Cowen ${ }^{55,56}$, R. Cross ${ }^{48}$, C. Dappen ${ }^{1}$, P. Dave ${ }^{6}$, C. De Clercq ${ }^{13}$, J. J. DeLaunay ${ }^{56}$, H. Dembinski ${ }^{42}$, K. Deoskar $^{50}$, S. De Ridder ${ }^{29}$, A. Desai ${ }^{38}$, P. Desiati ${ }^{38}$, K. D. de Vries ${ }^{13}$, G. de Wasseige ${ }^{13}$, M. de With ${ }^{10}$, T. DeYoung $^{24}$, S. Dharani $^{1}$, A. Diaz ${ }^{15}$, J. C. Díaz-Vélez ${ }^{38}$, M. Dittmer ${ }^{41}$, H. Dujmovic ${ }^{31}$, M. Dunkman ${ }^{56}$, M. A. DuVernois ${ }^{38}$, E. Dvorak ${ }^{46}$, T. Ehrhardt ${ }^{39}$, P. Eller $^{27}$, R. Engel ${ }^{31,}{ }^{32}$, H. Erpenbeck ${ }^{1}$, J. Evans ${ }^{19}$, P. A. Evenson ${ }^{42}$, K. L. Fan ${ }^{19}$, A. R. Fazely ${ }^{7}$, S. Fiedlschuster ${ }^{26}$, A. T. Fienberg ${ }^{56}$, K. Filimonov ${ }^{8}$, C. Finley ${ }^{50}$, L. Fischer ${ }^{59}$, D. Fox ${ }^{55}$, A. Franckowiak ${ }^{11,59}$, E. Friedman ${ }^{19}$, A. Fritz ${ }^{39}$, P. Fürst ${ }^{1}$, T. K. Gaisser ${ }^{42}$, J. Gallagher $^{37}$, E. Ganster ${ }^{1}$, A. Garcia ${ }^{14}$, S. Garrappa ${ }^{59}$, L. Gerhardt ${ }^{9}$, A. Ghadimi ${ }^{54}$, C. Glaser ${ }^{57}$, T. Glauch ${ }^{27}$, T. Glüsenkamp ${ }^{26}$, A. Goldschmidt $^{9}$, J. G. Gonzalez ${ }^{42}$, S. Goswami ${ }^{54}$, D. Grant ${ }^{24}$, T. Grégoire ${ }^{56}$, S. Griswold ${ }^{48}$, M. Gündüz ${ }^{11}$, C. Günther ${ }^{1}$, C. Haack ${ }^{27}$,

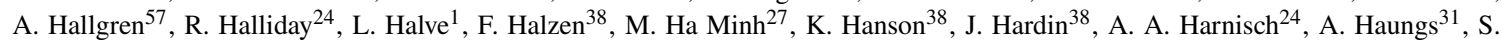

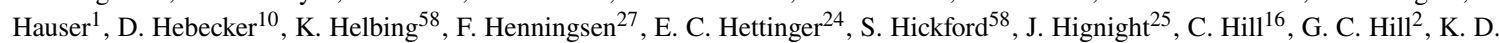

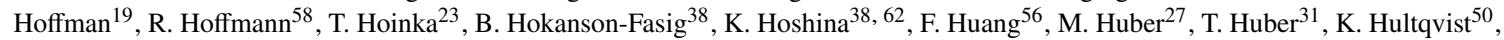
M. Hünnefeld ${ }^{23}$, R. Hussain ${ }^{38}$, S. In ${ }^{52}$, N. Iovine ${ }^{12}$, A. Ishihara ${ }^{16}$, M. Jansson ${ }^{50}$, G. S. Japaridze ${ }^{5}$, M. Jeong ${ }^{52}$, B. J. P. Jones ${ }^{4}$, D. Kang ${ }^{31}$, W. Kang ${ }^{52}$, X. Kang ${ }^{45}$, A. Kappes ${ }^{41}$, D. Kappesser ${ }^{39}$, T. Karg ${ }^{59}$, M. Karl ${ }^{27}$, A. Karle ${ }^{38}$, U. Katz ${ }^{26}$, M. Kauer ${ }^{38}$, M. Kellermann ${ }^{1}$, J. L. Kelley $^{38}$, A. Kheirandish ${ }^{56}$, K. Kin ${ }^{16}$, T. Kintscher ${ }^{59}$, J. Kiryluk ${ }^{51}$, S. R. Klein ${ }^{8,9}$, R. Koirala ${ }^{42}$, H. Kolanoski ${ }^{10}$, T. Kontrimas ${ }^{27}$, L. Köpke $^{39}$, C. Kopper ${ }^{24}$, S. Kopper ${ }^{54}$, D. J. Koskinen ${ }^{22}$, P. Koundal ${ }^{31}$, M. Kovacevich ${ }^{45}$, M. Kowalski ${ }^{10,59}$, T. Kozynets ${ }^{22}$, E. Kun ${ }^{11}$, N. Kurahashi ${ }^{45}$, N. Lad ${ }^{59}$, C. Lagunas Gualda ${ }^{59}$, J. L. Lanfranchi ${ }^{56}$, M. J. Larson ${ }^{19}$, F. Lauber ${ }^{58}$, J. P. Lazar ${ }^{14,38}$, J. W. Lee ${ }^{52}$, K. Leonard $^{38}$, A. Leszczyńska ${ }^{32}$, Y. Li ${ }^{56}$, M. Lincetto ${ }^{11}$, Q. R. Liu ${ }^{38}$, M. Liubarska ${ }^{25}$, E. Lohfink ${ }^{39}$, C. J. Lozano Mariscal ${ }^{41}$, L. Lu ${ }^{38}$, F. Lucarelli ${ }^{28}$, A. Ludwig ${ }^{24,35}$, W. Luszczak ${ }^{38}$, Y. Lyu ${ }^{8,9}$, W. Y. Ma ${ }^{59}$, J. Madsen ${ }^{38}$, K. B. M. Mahn ${ }^{24}$, Y. Makino ${ }^{38}$, S. Mancina ${ }^{38}$,

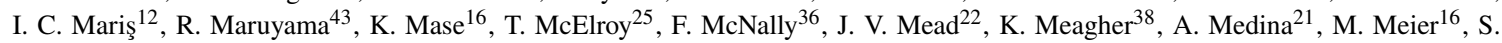
Meighen-Berger $^{27}$, J. Micallef ${ }^{24}$, D. Mockler ${ }^{12}$, T. Montaruli ${ }^{28}$, R. W. Moore ${ }^{25}$, R. Morse ${ }^{38}$, M. Moulai ${ }^{15}$, R. Naab ${ }^{59}$, R. Nagai ${ }^{16}$, U. Naumann ${ }^{58}$, J. Necker ${ }^{59}$, L. V. Nguyễn ${ }^{24}$, H. Niederhausen ${ }^{27}$, M. U. Nisa ${ }^{24}$, S. C. Nowicki ${ }^{24}$, D. R. Nygren ${ }^{9}$, A. Obertacke Pollmann ${ }^{58}$, M. Oehler ${ }^{31}$, A. Olivas ${ }^{19}$, E. O'Sullivan ${ }^{57}$, H. Pandya ${ }^{42}$, D. V. Pankova ${ }^{56}$, N. Park ${ }^{33}$, G. K. Parker ${ }^{4}$, E. N. Paudel ${ }^{42}$, L. Paul ${ }^{40}$, C. Pérez de los $\operatorname{Heros}^{57}$, L. Peters ${ }^{1}$, J. Peterson ${ }^{38}$, S. Philippen ${ }^{1}$, D. Pieloth ${ }^{23}$, S. Pieper ${ }^{58}$, M. Pittermann ${ }^{32}$, A. Pizzuto ${ }^{38}$, M. Plum ${ }^{40}$, Y. Popovych $^{39}$, A. Porcelli2 ${ }^{29}$, M. Prado Rodriguez ${ }^{38}$, P. B. Price ${ }^{8}$, B. Pries ${ }^{24}$, G. T. Przybylski ${ }^{9}$, C. Raab ${ }^{12}$, A. Raissi ${ }^{18}$, M. Rameez ${ }^{22}$, K. Rawlins $^{3}$, I. C. Rea ${ }^{27}$, A. Rehman ${ }^{42}$, P. Reichherzer ${ }^{11}$, R. Reimann ${ }^{1}$, G. Renzi ${ }^{12}$, E. Resconi ${ }^{27}$, S. Reusch ${ }^{59}$, W. Rhode ${ }^{23}$, M. Richman ${ }^{45}$, B. Riedel ${ }^{38}$, E. J. Roberts ${ }^{2}$, S. Robertson ${ }^{8,9}$, G. Roellinghoff ${ }^{52}$, M. Rongen ${ }^{39}$, C. Rott ${ }^{49}, 52$, T. Ruhe ${ }^{23}$, D. Ryckbosch ${ }^{29}$, D. Rysewyk $\mathrm{Cantu}^{24}$, I. Safa ${ }^{14,38}$, J. Saffer ${ }^{32}$, S. E. Sanchez Herrera ${ }^{24}$, A. Sandrock ${ }^{23}$, J. Sandroos ${ }^{39}$, M. Santander ${ }^{54}$, S. Sarkar ${ }^{44}$, S. Sarkar ${ }^{25}$, K. Satalecka $^{59}$, M. Scharf ${ }^{1}$, M. Schaufel ${ }^{1}$, H. Schieler ${ }^{31}$, S. Schindler ${ }^{26}$, P. Schlunder ${ }^{23}$, T. Schmidt ${ }^{19}$, A. Schneider ${ }^{38}$, J. Schneider ${ }^{26}$, F. G. Schröder ${ }^{31,42}$, L. Schumacher ${ }^{27}$, G. Schwefer ${ }^{1}$, S. Sclafani ${ }^{45}$, D. Seckel ${ }^{42}$, S. Seunarine ${ }^{47}$, A. Sharma ${ }^{57}$, S. Shefali ${ }^{32}$, M. Silva $^{38}$, B. Skrzypek ${ }^{14}$, B. Smithers ${ }^{4}$, R. Snihur ${ }^{38}$, J. Soedingrekso ${ }^{23}$, D. Soldin ${ }^{42}$, C. Spannfellner ${ }^{27}$, G. M. Spiczak ${ }^{47}$, C. Spiering ${ }^{59,}$, ${ }^{2}$, J. Stachurska $^{59}$, M. Stamatikos ${ }^{21}$, T. Stanev ${ }^{42}$, R. Stein ${ }^{59}$, J. Stettner ${ }^{1}$, A. Steuer ${ }^{39}$, T. Stezelberger ${ }^{9}$, T. Stürwald ${ }^{58}$, T. Stuttard ${ }^{22}$, G. W. Sullivan $^{19}$, I. Taboada ${ }^{6}$, F. Tenholt ${ }^{11}$, S. Ter-Antonyan ${ }^{7}$, S. Tilav ${ }^{42}$, F. Tischbein ${ }^{1}$, K. Tollefson $^{24}$, L. Tomankova ${ }^{11}$, C. Tönnis ${ }^{53}$, S. Toscano $^{12}$, D. Tosi ${ }^{38}$, A. Trettin ${ }^{59}$, M. Tselengidou ${ }^{26}$, C. F. Tung ${ }^{6}$, A. Turcati2 ${ }^{27}$, R. Turcotte ${ }^{31}$, C. F. Turley ${ }^{56}$, J. P. Twagirayezu ${ }^{24}$, B. $\mathrm{Ty}^{38}$, M. A. Unland Elorrieta ${ }^{41}$, N. Valtonen-Mattila ${ }^{57}$, J. Vandenbroucke ${ }^{38}$, N. van Eijndhoven ${ }^{13}$, D. Vannerom ${ }^{15}$, J. van Santen ${ }^{59}, \mathrm{~S}$.

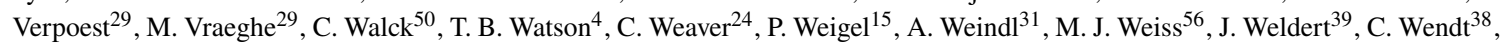
J. Werthebach ${ }^{23}$, M. Weyrauch ${ }^{32}$, N. Whitehorn ${ }^{24,}{ }^{35}$, C. H. Wiebusch ${ }^{1}$, D. R. Williams ${ }^{54}$, M. Wolf ${ }^{27}$, K. Woschnagg ${ }^{8}$, G. Wrede ${ }^{26}$, J. Wulff ${ }^{11}$, X. W. Xu ${ }^{7}$, Y. Xu ${ }^{51}$, J. P. Yanez ${ }^{25}$, S. Yoshida ${ }^{16}$, S. Yu ${ }^{24}$, T. Yuan ${ }^{38}$, Z. Zhang ${ }^{51}$

${ }^{1}$ III. Physikalisches Institut, RWTH Aachen University, D-52056 Aachen, Germany

${ }^{2}$ Department of Physics, University of Adelaide, Adelaide, 5005, Australia

${ }^{3}$ Dept. of Physics and Astronomy, University of Alaska Anchorage, 3211 Providence Dr., Anchorage, AK 99508, USA

${ }^{4}$ Dept. of Physics, University of Texas at Arlington, 502 Yates St., Science Hall Rm 108, Box 19059, Arlington, TX 76019, USA

${ }^{5}$ CTSPS, Clark-Atlanta University, Atlanta, GA 30314, USA

${ }^{6}$ School of Physics and Center for Relativistic Astrophysics, Georgia Institute of Technology, Atlanta, GA 30332, USA

${ }^{7}$ Dept. of Physics, Southern University, Baton Rouge, LA 70813, USA

${ }^{8}$ Dept. of Physics, University of California, Berkeley, CA 94720, USA

${ }^{9}$ Lawrence Berkeley National Laboratory, Berkeley, CA 94720, USA

${ }^{10}$ Institut für Physik, Humboldt-Universität zu Berlin, D-12489 Berlin, Germany

${ }^{11}$ Fakultät für Physik \& Astronomie, Ruhr-Universität Bochum, D-44780 Bochum, Germany

${ }^{12}$ Université Libre de Bruxelles, Science Faculty CP230, B-1050 Brussels, Belgium

${ }^{13}$ Vrije Universiteit Brussel (VUB), Dienst ELEM, B-1050 Brussels, Belgium

${ }^{14}$ Department of Physics and Laboratory for Particle Physics and Cosmology, Harvard University, Cambridge, MA 02138, USA

${ }^{15}$ Dept. of Physics, Massachusetts Institute of Technology, Cambridge, MA 02139, USA 
16 Dept. of Physics and Institute for Global Prominent Research, Chiba University, Chiba 263-8522, Japan

17 Department of Physics, Loyola University Chicago, Chicago, IL 60660, USA

18 Dept. of Physics and Astronomy, University of Canterbury, Private Bag 4800, Christchurch, New Zealand

19 Dept. of Physics, University of Maryland, College Park, MD 20742, USA

${ }^{20}$ Dept. of Astronomy, Ohio State University, Columbus, OH 43210, USA

21 Dept. of Physics and Center for Cosmology and Astro-Particle Physics, Ohio State University, Columbus, OH 43210, USA

22 Niels Bohr Institute, University of Copenhagen, DK-2100 Copenhagen, Denmark

23 Dept. of Physics, TU Dortmund University, D-44221 Dortmund, Germany

${ }^{24}$ Dept. of Physics and Astronomy, Michigan State University, East Lansing, MI 48824, USA

25 Dept. of Physics, University of Alberta, Edmonton, Alberta, Canada T6G 2E1

${ }^{26}$ Erlangen Centre for Astroparticle Physics, Friedrich-Alexander-Universität Erlangen-Nürnberg, D-91058 Erlangen, Germany

${ }^{27}$ Physik-department, Technische Universität München, D-85748 Garching, Germany

28 Département de physique nucléaire et corpusculaire, Université de Genève, CH-1211 Genève, Switzerland

${ }^{29}$ Dept. of Physics and Astronomy, University of Gent, B-9000 Gent, Belgium

${ }^{30}$ Dept. of Physics and Astronomy, University of California, Irvine, CA 92697, USA

${ }^{31}$ Karlsruhe Institute of Technology, Institute for Astroparticle Physics, D-76021 Karlsruhe, Germany

${ }^{32}$ Karlsruhe Institute of Technology, Institute of Experimental Particle Physics, D-76021 Karlsruhe, Germany

33 Dept. of Physics, Engineering Physics, and Astronomy, Queen's University, Kingston, ON K7L 3N6, Canada

34 Dept. of Physics and Astronomy, University of Kansas, Lawrence, KS 66045, USA

35 Department of Physics and Astronomy, UCLA, Los Angeles, CA 90095, USA

36 Department of Physics, Mercer University, Macon, GA 31207-0001, USA

${ }^{37}$ Dept. of Astronomy, University of Wisconsin-Madison, Madison, WI 53706, USA

${ }^{38}$ Dept. of Physics and Wisconsin IceCube Particle Astrophysics Center, University of Wisconsin-Madison, Madison, WI 53706, USA

${ }^{39}$ Institute of Physics, University of Mainz, Staudinger Weg 7, D-55099 Mainz, Germany

40 Department of Physics, Marquette University, Milwaukee, WI, 53201, USA

${ }^{41}$ Institut für Kernphysik, Westfälische Wilhelms-Universität Münster, D-48149 Münster, Germany

42 Bartol Research Institute and Dept. of Physics and Astronomy, University of Delaware, Newark, DE 19716, USA

43 Dept. of Physics, Yale University, New Haven, CT 06520, USA

44 Dept. of Physics, University of Oxford, Parks Road, Oxford OX1 3PU, UK

45 Dept. of Physics, Drexel University, 3141 Chestnut Street, Philadelphia, PA 19104, USA

46 Physics Department, South Dakota School of Mines and Technology, Rapid City, SD 57701, USA

47 Dept. of Physics, University of Wisconsin, River Falls, WI 54022, USA

48 Dept. of Physics and Astronomy, University of Rochester, Rochester, NY 14627, USA

49 Department of Physics and Astronomy, University of Utah, Salt Lake City, UT 84112, USA

${ }^{50}$ Oskar Klein Centre and Dept. of Physics, Stockholm University, SE-10691 Stockholm, Sweden

${ }^{51}$ Dept. of Physics and Astronomy, Stony Brook University, Stony Brook, NY 11794-3800, USA

52 Dept. of Physics, Sungkyunkwan University, Suwon 16419, Korea

53 Institute of Basic Science, Sungkyunkwan University, Suwon 16419, Korea

54 Dept. of Physics and Astronomy, University of Alabama, Tuscaloosa, AL 35487, USA

55 Dept. of Astronomy and Astrophysics, Pennsylvania State University, University Park, PA 16802, USA

56 Dept. of Physics, Pennsylvania State University, University Park, PA 16802, USA

${ }^{57}$ Dept. of Physics and Astronomy, Uppsala University, Box 516, S-75120 Uppsala, Sweden

58 Dept. of Physics, University of Wuppertal, D-42119 Wuppertal, Germany

59 DESY, D-15738 Zeuthen, Germany

${ }^{60}$ Università di Padova, I-35131 Padova, Italy

61 National Research Nuclear University, Moscow Engineering Physics Institute (MEPhI), Moscow 115409, Russia

${ }^{62}$ Earthquake Research Institute, University of Tokyo, Bunkyo, Tokyo 113-0032, Japan

\section{Acknowledgements}

USA - U.S. National Science Foundation-Office of Polar Programs, U.S. National Science Foundation-Physics Division, U.S. National Science Foundation-EPSCoR, Wisconsin Alumni Research Foundation, Center for High Throughput Computing (CHTC) at the University of Wisconsin-Madison, Open Science Grid (OSG), Extreme Science and Engineering Discovery Environment (XSEDE), Frontera computing project at the Texas Advanced Computing Center, U.S. Department of Energy-National Energy Research Scientific Computing Center, Particle astrophysics research computing center at the University of Maryland, Institute for Cyber-Enabled Research at Michigan State University, and Astroparticle physics computational facility at Marquette University; Belgium - Funds for Scientific Research (FRS-FNRS and FWO), FWO Odysseus and Big Science programmes, and Belgian Federal Science Policy Office (Belspo); Germany - Bundesministerium für Bildung und Forschung (BMBF), Deutsche Forschungsgemeinschaft (DFG), Helmholtz Alliance for Astroparticle Physics (HAP), Initiative and Networking Fund of the Helmholtz Association, Deutsches Elektronen Synchrotron (DESY), and High Performance Computing cluster of the RWTH Aachen; Sweden - Swedish Research Council, Swedish Polar Research Secretariat, Swedish National Infrastructure for Computing (SNIC), and Knut and Alice Wallenberg Foundation; Australia - Australian 
Research Council; Canada - Natural Sciences and Engineering Research Council of Canada, Calcul Québec, Compute Ontario, Canada Foundation for Innovation, WestGrid, and Compute Canada; Denmark - Villum Fonden and Carlsberg Foundation; New Zealand Marsden Fund; Japan - Japan Society for Promotion of Science (JSPS) and Institute for Global Prominent Research (IGPR) of Chiba University; Korea - National Research Foundation of Korea (NRF); Switzerland - Swiss National Science Foundation (SNSF); United Kingdom - Department of Physics, University of Oxford. 